\title{
Plant buffering against the high-light stress- induced accumulation of CsGA2ox8 transcripts via alternative splicing to finely tune gibberellin levels and maintain hypocotyl elongation
}

\author{
Bin Liư ${ }^{1,2}$, Shuo Zhao ${ }^{1}$, Pengli Li', Yilu Yin ${ }^{1}$, Qingliang Niu', Jinqiang Yan ${ }^{3,4}$ and Danfeng Huang ${ }^{1}$
}

\begin{abstract}
In plants, alternative splicing (AS) is markedly induced in response to environmental stresses, but it is unclear why plants generate multiple transcripts under stress conditions. In this study, RNA-seq was performed to identify AS events in cucumber seedlings grown under different light intensities. We identified a novel transcript of the gibberellin (GA)-deactivating enzyme Gibberellin 2-beta-dioxygenase 8 (CsGA20x8). Compared with canonical CsGA2ox8.1, the CsGA20x8.2 isoform presented intron retention between the second and third exons. Functional analysis proved that the transcript of CsGA20x8.1 but not CsGA20x8.2 played a role in the deactivation of bioactive GAs. Moreover, expression analysis demonstrated that both transcripts were upregulated by increased light intensity, but the expression level of CSGA20x8.1 increased slowly when the light intensity was $>400 \mu \mathrm{mol} \cdot \mathrm{m}^{-2} \cdot \mathrm{s}^{-1} \mathrm{PPFD}$ (photosynthetic photon flux density), while the CsGA20x8.2 transcript levels increased rapidly when the light intensity was $>200 \mu \mathrm{mol} \cdot \mathrm{m}^{-2} \cdot \mathrm{s}^{-1}$ PPFD. Our findings provide evidence that plants might finely tune their GA levels by buffering against the normal transcripts of CSGA20x8 through AS.
\end{abstract}

\section{Introduction}

Gibberellins (GAs) are known to be the dominant hormone governing hypocotyl elongation ${ }^{1,2}$. GAs regulate cell expansion, thus causing changes in hypocotyl length or plant height via the GA signaling pathway. GA-GID1DELLA is a widely accepted signaling module through which bioactive GAs regulate plant growth and development $^{2}$. For example, GIBBERELLIN INSENSITIVE DWARF 1 (GID1)-inactivation mutants show reduced plant height ${ }^{3,4}$, and substitution of proline to leucine in

Correspondence: Jinqiang Yan (yan.jinqiang@163.com) or

Danfeng Huang (hdf@sjtu.edu.cn)

${ }^{1}$ School of Agriculture and Biology, Shanghai Jiao Tong University, Key

Laboratory of Urban Agriculture (South), Ministry of Agriculture, Dongchuan Road, Shanghai 200240, China

${ }^{2}$ Department of Plant Genomics, Centre for Research in Agricultural Genomics (CRAG), CSIC-IRTA-UAB-UB, Bellaterra 08193, Spain

Full list of author information is available at the end of the article
DS-3, which encodes a DELLA protein, results in semidwarf oilseed rape plants 5 .

Light is among the most essential factors for plant growth and development ${ }^{6}$. Seedlings grown under dark conditions are characterized by relatively long hypocotyls compared with those grown under light conditions ${ }^{7,8}$. One of the reasons for this is that under light conditions, the GA level decreases, while under dark or shaded conditions, the GA level increases ${ }^{9-11}$. Most related studies have focused mainly on low-light (shading)-induced GA biosynthesis for signaling ${ }^{12}$, and only a few studies have shown high-light-induced excessive GA deactivation. However, it remains unknown how plants respond to high-light stress and finely tune their GA levels to maintain growth.

Alternative splicing (AS) is a widespread mechanism in eukaryotes in which different protein variants are generated from a single gene via different combinations of

\section{(c) The Author(s) 2020}

(c) (i) Open Access This article is licensed under a Creative Commons Attribution 4.0 International License, which permits use, sharing, adaptation, distribution and reproduction c. in any medium or format, as long as you give appropriate credit to the original author(s) and the source, provide a link to the Creative Commons license, and indicate if changes were made. The images or other third party material in this article are included in the article's Creative Commons license, unless indicated otherwise in a credit line to the material. If material is not included in the article's Creative Commons license and your intended use is not permitted by statutory regulation or exceeds the permitted use, you will need to obtain permission directly from the copyright holder. To view a copy of this license, visit http://creativecommons.org/licenses/by/4.0/. 
splicing sites. AS is a prevalent approach in which plants respond to environmental stress, but it is counterintuitive why plants would invest in protein synthesis under conditions of declining energy supplies ${ }^{13,14}$. Chaudhary et al. proposed that, under stress conditions, plants buffer against normal protein synthesis levels via AS to decrease the translation of a significant portion of the transcriptome and produce the protein isoforms necessary for adaptation to stresses; however, the mechanisms of this process are not yet known ${ }^{14}$.

In this study, we performed RNA-seq to identify differentially expressed genes and AS events in cucumber (Cucumis sativus L.) seedlings grown under different light intensities. We focused on GA-related genes and found a novel transcript of Gibberellin 2-beta-dioxygenase (CsGA2ox8), CsGA2ox8.2, with intron retention (IR) between the second and third exons. Gibberellin 2-betadioxygenases compose a family whose members can catalyze the deactivation of bioactive GAs or their precursors $^{15}$. Most studies have shown that increasing transcripts of GA2oxs can reduce plant height by regulating endogenous bioactive GA levels ${ }^{15-20}$. We found that under high-light stress, cucumber seedlings tended to generate more nonfunctional CsGA2ox8.2 but not functional canonical CsGA2ox8.1. In other words, the plants buffered against normal transcript levels of $C s G A 20 x 8$ via AS to limit the inactivation process of GAs under highlight stress, and this strategy may allow seedlings to maintain a certain GA level and sustain hypocotyl elongation.

\section{Results and discussion}

\section{CsGA20x8 expression is upregulated in response to increasing light intensity}

We recorded the changes in cucumber hypocotyl lengths after the cotyledons emerged from the soil under treatments involving two light intensities: $\mathrm{X} 1$ $\left(180 \mu \mathrm{mol} \cdot \mathrm{m}^{-2} \cdot \mathrm{s}^{-1} \quad\right.$ PPFD $)$ and X2 $\left(40 \mu \mathrm{mol} \cdot \mathrm{m}^{-2} \cdot \mathrm{s}^{-1}\right.$ PPFD). The hypocotyl lengths of seedlings grown under treatment $\mathrm{X} 1$ were shorter than those grown under treatment X2 (Fig. 1a), and a significant difference was observed after $40 \mathrm{~h}$ of light treatment (Fig. 1b). To investigate the molecular differences of seedlings grown under the two light conditions, a transcriptome analysis was performed. In total, 279 upregulated and 321 downregulated genes were identified between the two treatments (Fig. 1c, Supplementary Table S1). In this study, we focused on GA-related genes because bioactive GAs have a large effect on hypocotyl elongation in cucumber $^{1}$. We found that $C s G A 20 x 8$, a gene whose product catalyzes the deactivation of bioactive GAs, was significantly upregulated in treatment $\mathrm{X} 1$ compared to treatment X2. This expression difference was confirmed by quantitative reverse-transcription PCR (qRT-PCR) (Fig. 1d). These results suggested that the expression of CsGA20x8 might be affected by light intensity. To test this hypothesis, we treated young cucumber seedlings with seven different light intensities (40, 80, 120, 160, 200, 240, and $280 \mu \mathrm{mol} \cdot \mathrm{m}^{-2} \cdot \mathrm{s}^{-1}$ PPFD) and quantified the expression of CsGA2ox8 in seedlings grown under these conditions. As shown in Fig. 1e, the transcript level of CsGA2ox8 increased with increasing light intensity. Pioneering studies have shown that light modulates photomorphogenesis-related hypocotyl elongation mainly by interacting with GAs, and many genes are involved in this light-GA system ${ }^{10}$. Our results suggested that the expression of the GA deactivation factor CsGA2ox8 was upregulated by increased light levels, which might be involved in light-regulated hypocotyl elongation.

\section{Differential AS (DAS) analysis for identifying novel transcripts of CsGA2ox8}

AS is a prevalent approach used by plants in response to stress and involves the generation of multiple transcripts from the same gene ${ }^{21}$. In this study, five types of AS events, namely, RIs (retained introns), A5SSs (alternative $5^{\prime}$ splice sites), A3SS (alternative $3^{\prime}$ splice sites), MXEs (mutually exclusive exons), and SEs (skipped exons), were identified by RNA-seq analysis (Fig. 2a, Supplementary Table S2). Among them, SEs and RIs accounted for more than $25 \%$ of all AS events (Fig. 2b), which is similar to that reported in Arabidopsis ${ }^{22,23}$.

Among all RI events, we found a novel transcript of CsGA2ox8 with an IR between the second and third exons. The original transcript of $C s G A 2 o x 8$ was defined as CsGA2ox8.1, and the isoform with IR was defined as CsGA20x8.2 (Fig. 2c). To validate the existence of CsGA2ox8.2, the coding regions of CsGA2ox8 of plants under treatment X2 were amplified and then sequenced. Two distinct bands were observed after RT-PCR-based amplification (Fig. 2c). After sequencing, the two amplicons were found to be 1056 and 1655 bp in length (Fig. 2c). Sequence comparison showed the same result as that after RNA-seq, and a schematic diagram of this event is illustrated in Fig. 2c. RIs promoted the disruption of the open reading frame of $C s G A 20 x 8$, resulting in an mRNA with a premature stop codon (TGA), and leading to a truncated protein (Fig. 2c). Compared with other GA2ox proteins in Arabidopsis, the AtGA2ox7 and AtGA2ox8 proteins have a unique region ${ }^{15}$. Alignment of the amino acid sequences of AtGA2ox8 and CsGA2ox8.1 revealed that the CsGA2ox8.1 protein had a conserved motif (Fig. $2 \mathrm{~d}$, denoted by a red box), which may define the specificity of the reactions catalyzed by these enzymes ${ }^{15}$. The absence of amino acids in CsGA2ox8.2 is indicated using a purple line in Fig. 2d. 

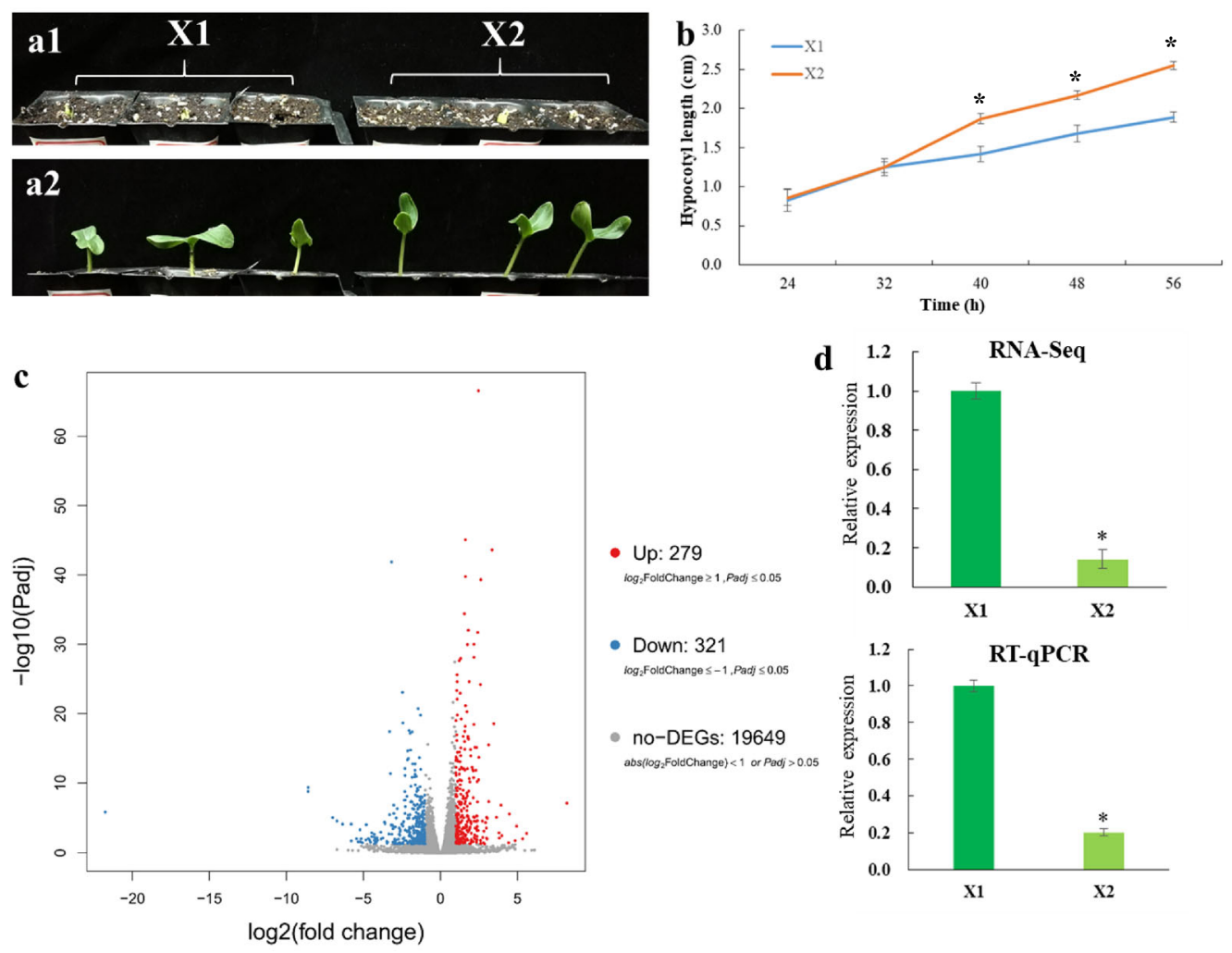

e

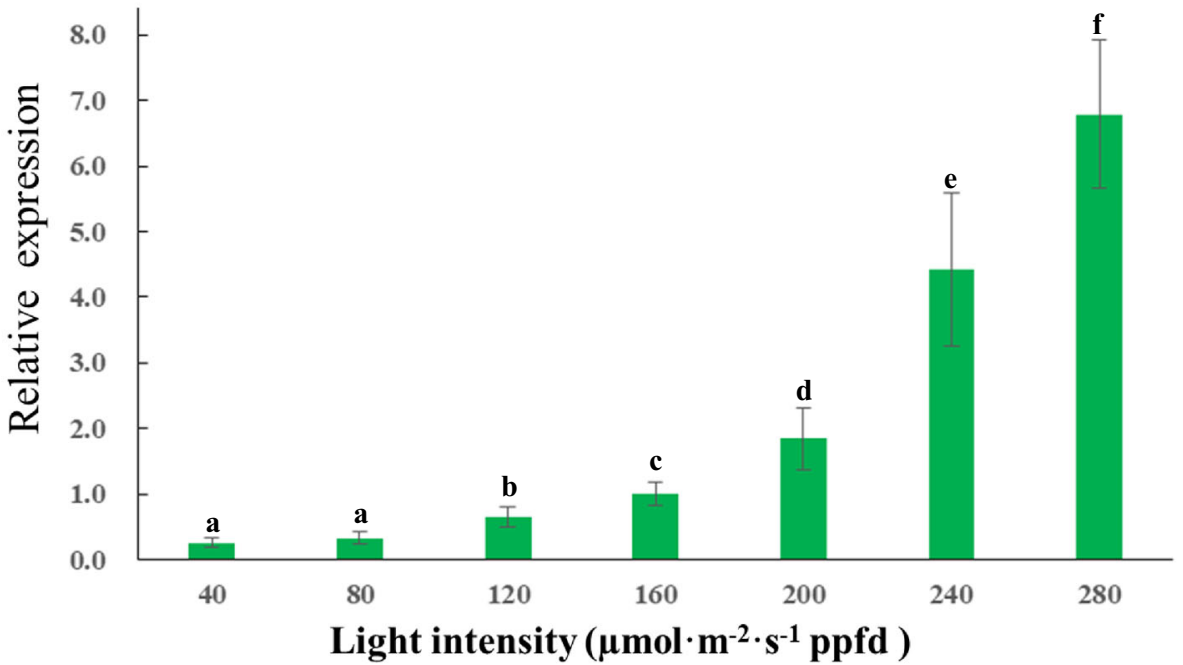

Fig. 1 Expression of CsGA2ox8 is positively regulated by light intensity. a Phenotypes of cucumber seedlings before (a1) and after (a2) treatment with fluorescent lights at two intensities $\left(X 1,180 \mu \mathrm{mol} \cdot \mathrm{m}^{-2} \cdot \mathrm{s}^{-1}\right.$ PPFD; $X 2,40 \mu \mathrm{mol} \cdot \mathrm{m}^{-2} \cdot \mathrm{s}^{-1}$ PPFD) for $56 \mathrm{~h}$. b Hypocotyl lengths of cucumber seedlings between the two treatments. The error bars indicate the standard deviations (SDs; $n=5)$. The asterisks indicate significant differences in hypocotyl lengths between X1 and X2 $(P<0.05)$. c Volcano plot of the transcriptome between X1-and X2-treated cucumber seedlings. d Quantitative reverse-transcription PCR results (three replications) validating the different expressions of CsGA20x8 between the two treatments. The asterisks indicate significant differences in gene expression between X1 and X2 $(P<0.05)$. e Relative expression of CsGA2ox8 under different light intensities. The lowercase letters indicate that CsGA20x8 transcription was modified by light intensity $(P<0.05$, three replications) 
a

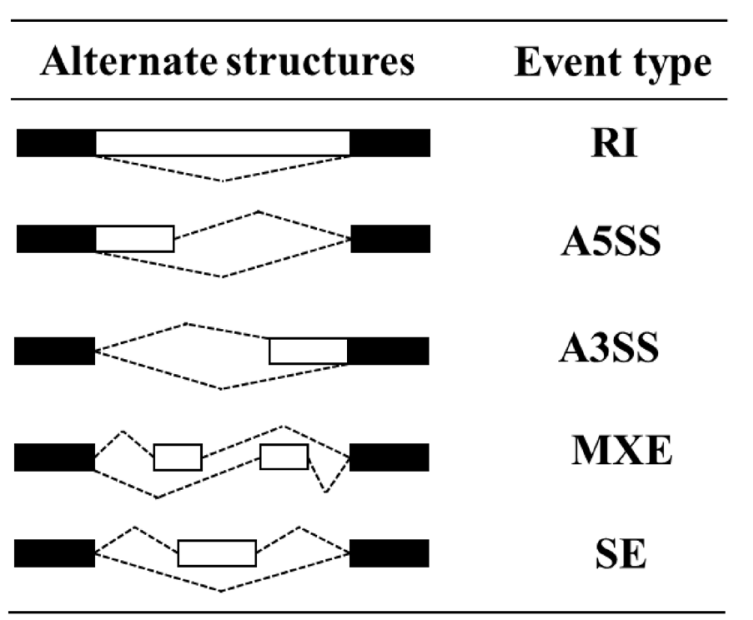

b

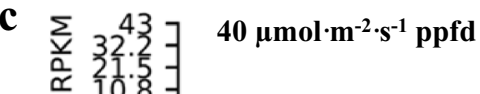

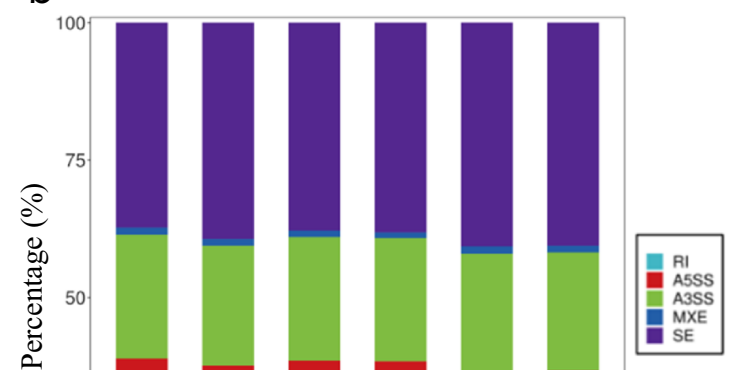

$\sum^{2} \quad 180 \mu \mathrm{mol} \cdot \mathrm{m}^{-2} \cdot \mathrm{s}^{-1} \mathbf{p p f d}$

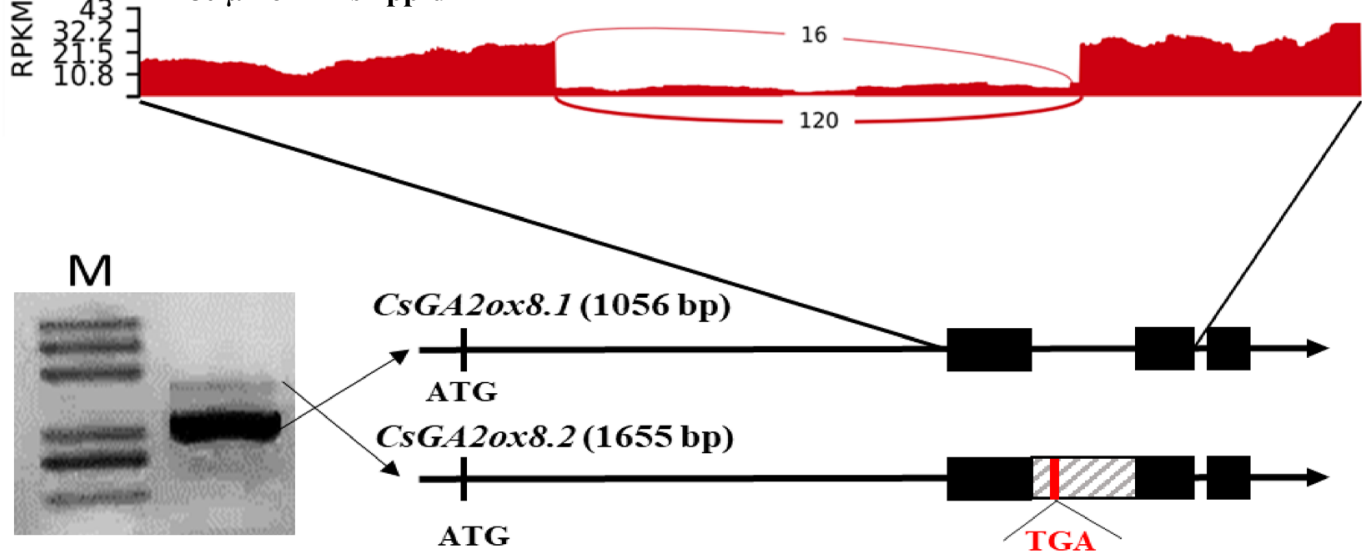

d

AtGA20x8

CsGA2ox8.1

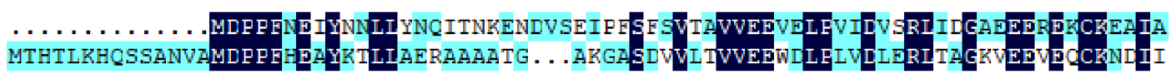

Consensus

mappe $y$ y

X1_1 X1_2 X1_3 X2_1 X2_2 X2_3

AtGA20x8

CsGA20x8.1

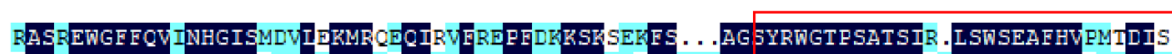

Consensus

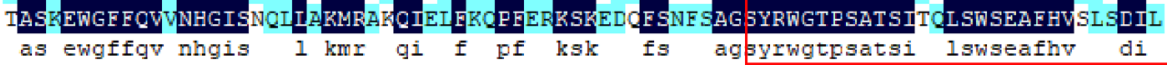

AtGA20x8

CsGA20x8.1

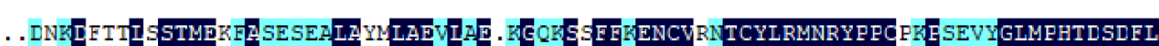

Consensus

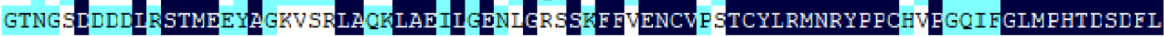

$$
\text { d l stme a la lae le g } g \text { fi encv teylrmnrype p glmphtdsdfl }
$$

AtGA20x8

CsGA2ox8.1

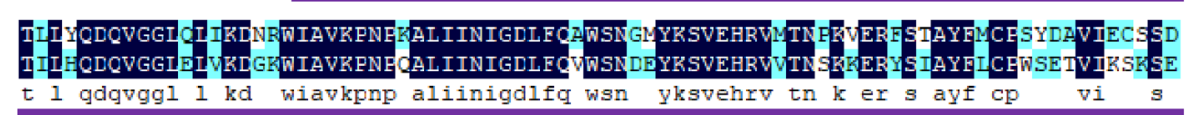

AtGA20x8

CsGA20x8.1

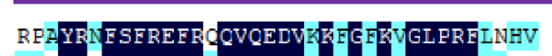

Consensus

PGVYRRESFREFRNQVQEDVRGYGYIIGLPREVI. .

yr fsfrefr qugedv $\mathrm{k} g \mathrm{k}$ glprf

Fig. 2 (See legend on next page.) 
(see figure on previous page)

Fig. 2 Analysis of differential alternative splicing (DAS) for the identification of novel transcripts of Gibberellin 2-beta-dioxygenase 8 (CsGA2ox8). a Identification of five AS events in the RNA-seq datasets of cucumber seedlings grown under two light conditions. RI, retained intron; A5SS, alternative $5^{\prime}$ splice site; A3SS, alternative $3^{\prime}$ splice site; MXE, mutually exclusive exon; and SE, skipped exon. Black boxes, exons flanking AS events; white boxes, differentially included exons; dotted lines, sequences spliced together. b Stacked bars showing five AS events and their frequency in each replication under the two light conditions. $\mathbf{c}$ Validation of the intron retention (IR) of CSGA2Ox8 via reverse-transcription PCR (RTPCR). A termination codon, TGA (red line), was detected in the retained intron (gray box). M, Trans2K Plus DNA Marker. $\mathbf{d}$ Alignment of the amino acid sequences of AtGA20x8 and CsGA20x8.1. The unique conserved domain of GA20x8.1 is denoted by the red box above. Purple line, the lost amino acid region of CsGA20x8.2

\section{Overexpression of CsGA20x8.1 but not CsGA20x8.2 decreases GA levels and the length of the hypocotyl in Arabidopsis thaliana}

GA2ox genes function in reducing $\mathrm{GAs}^{15-20,24}$. Given that $C s G A 20 x 8.2$ lost the third and fourth exons from CsGA2ox8.1 (Fig. 2c, d), we questioned whether CsGA2ox8.2 still maintains the same function as that of CsGA2ox8.1. To verify this, CsGA2ox8.1 and CsGA2ox8.2 were overexpressed in Arabidopsis Col-0. CsGA2ox8.1 overexpression lines (OE8.1) had shorter hypocotyl lengths than Col-0 under both light and dark conditions (Fig. 3a-c). The RT-PCR results suggested that GA2ox8.1 was overexpressed in OE8.1 but not in Col-0 (Fig. 3d), suggesting that GA2ox8.1 was functional for hypocotyl elongation. However, the hypocotyl length of CsGA2ox8.2 overexpression lines (OE8.2) was not significantly different from that of Col-0 (Fig. $3 \mathrm{e}-\mathrm{g}$ ), even though the transcript level of GA2ox8.2 was as high as that of GA2ox8.1 (Figs. 3d, h). Next, the GA levels were quantified in the Col-0, OE8.1, and OE8.2 lines. Based on the GA synthetic pathway, GA2ox genes encoding enzymes that convert $\mathrm{GA}_{12}, \mathrm{GA}_{9}, \mathrm{GA}_{53}, \mathrm{GA}_{20}, \mathrm{GA}_{1}$, and $\mathrm{GA}_{4}$ to $\mathrm{GA}_{110}, \mathrm{GA}_{51}, \mathrm{GA}_{97}, \mathrm{GA}_{29}, \mathrm{GA}_{8}$, and $\mathrm{GA}_{34}$, respectively ${ }^{25,26}$ (Fig. 3i). In this study, we found that $\mathrm{GA}_{12}, \mathrm{GA}_{9}$, $\mathrm{GA}_{53}, \mathrm{GA}_{20}, \mathrm{GA}_{1}$, and $\mathrm{GA}_{4}$ significantly decreased in OE8.1 compared with Col-0, and no significant differences in GA levels were detected between OE8.2 and Col0 (Fig. 3i), suggesting that $C s G A 20 x 8.1$ is a functional transcript but that $C s G A 20 x 8.2$ is not.

AS is a prevalent approach in which plants respond to environmental stress. One hypothesis is that plants buffer against normal protein synthesis levels via AS to decrease the translation of a significant portion of the transcriptome under stress conditions ${ }^{14}$. Therefore, for future studies, it is important to know how CsGA2ox8.1 and CsGA2ox8.2 work at the protein level.

The homolog of CsGA2ox8 in Arabidopsis is AtGA2ox8, whose product functions in hydroxylating the $\mathrm{C}_{20}-\mathrm{GA}$ precursors $\mathrm{GA}_{12}$ and $\mathrm{GA}_{53}{ }^{15}$. In this study, consistent with the results from overexpression of AtGA2ox $8^{15}$, $\mathrm{GA}_{12}$ and $\mathrm{GA}_{53}$ levels dramatically decreased in OE8.1, suggesting that $C s G A 2 o x 8$ has a function similar to that of AtGA2ox8. However, we found that the content of $\mathrm{GA}_{9}$, $\mathrm{GA}_{20}, \mathrm{GA}_{1}$, and $\mathrm{GA}_{4}$ also decreased, which is inconsistent with the results of Schomburg et al. (2003), who reported that $\mathrm{GA}_{9}, \mathrm{GA}_{20}$, and $\mathrm{GA}_{4}$ levels increased in AtGA2ox8 overexpression lines ${ }^{15} \cdot \mathrm{GA}_{12}$ is the precursor of $\mathrm{GA}_{9}$ and $\mathrm{GA}_{4}$, and $\mathrm{GA}_{53}$ is the precursor of $\mathrm{GA}_{20}$ and $\mathrm{GA}_{1}{ }^{25}$. Therefore, it is possible that decreasing $\mathrm{GA}_{12}$ and $\mathrm{GA}_{53}$ levels would affect the synthesis of $\mathrm{GA}_{9}, \mathrm{GA}_{4}, \mathrm{GA}_{20}$, and $\mathrm{GA}_{1}$. Taken together, these results suggest that CsGA2ox8.1, rather than its CsGA2ox8.2 isoform, regulated hypocotyl elongation, and this regulation was achieved mostly via decreasing endogenous GA levels.

\section{Plants tend to generate nonfunctional CsGA20x8.2 but not functional CsGA20x8.1 under high-light stress}

As one type of AS, IR mostly results in the production of nonfunctional transcripts carrying premature termination codons targeted for degradation via the nonsensemediated mRNA decay pathway ${ }^{27,28}$. However, it is unclear why plants perform AS under stress conditions. Given that CsGA2ox8.2 was detected under relatively high-light conditions and that CsGA2ox8.2 seemed to be a nonfunctional transcript, we hypothesized that CsGA2ox8.2 was generated for buffering against normal CsGA2ox8.1 transcripts under high-light stress to finely tune GA levels and maintain plant growth. Chaudhary et al. posited the same idea and proposed that, under stress conditions, plants buffer against normal protein synthesis levels via AS to decrease translation of a significant portion of the transcriptome, but the mechanisms of this process have not yet been elucidated ${ }^{14}$.

To test our hypothesis, the relative abundance of the two mRNA forms of CsGA2ox8 in plants under 50, 100, 150, 200, 400, 800, and $1200 \mu \mathrm{mol} \cdot \mathrm{m}^{-2} \cdot \mathrm{s}^{-1} \mathrm{PPFD}$ was analyzed by RT-PCR. The results showed that both transcripts were positively regulated by light, and CsGA20x8.1 was the dominant transcript under all light conditions (Fig. 4a). However, the expression level of CsGA2ox8.1 increased slowly when the light intensity was $>400 \mu \mathrm{mol} \cdot \mathrm{m}^{-2} \cdot \mathrm{s}^{-1}$ PPFD, while that of CsGA2ox8.2 increased rapidly when the light intensity was $>200 \mu \mathrm{mol} \cdot \mathrm{m}^{-2} \cdot \mathrm{s}^{-1}$ PPFD (Fig. 4a). Furthermore, the inclusion/exclusion (In/Ex) ratio (CsGA2ox8.2/ CsGA2ox8.1) significantly increased when the light intensity was $>400 \mu \mathrm{mol} \cdot \mathrm{m}^{-2} \cdot \mathrm{s}^{-1}$ PPFD (Fig. $4 \mathrm{~b}$ ). In other words, the CsGA2ox8.2 expression level was negligible 

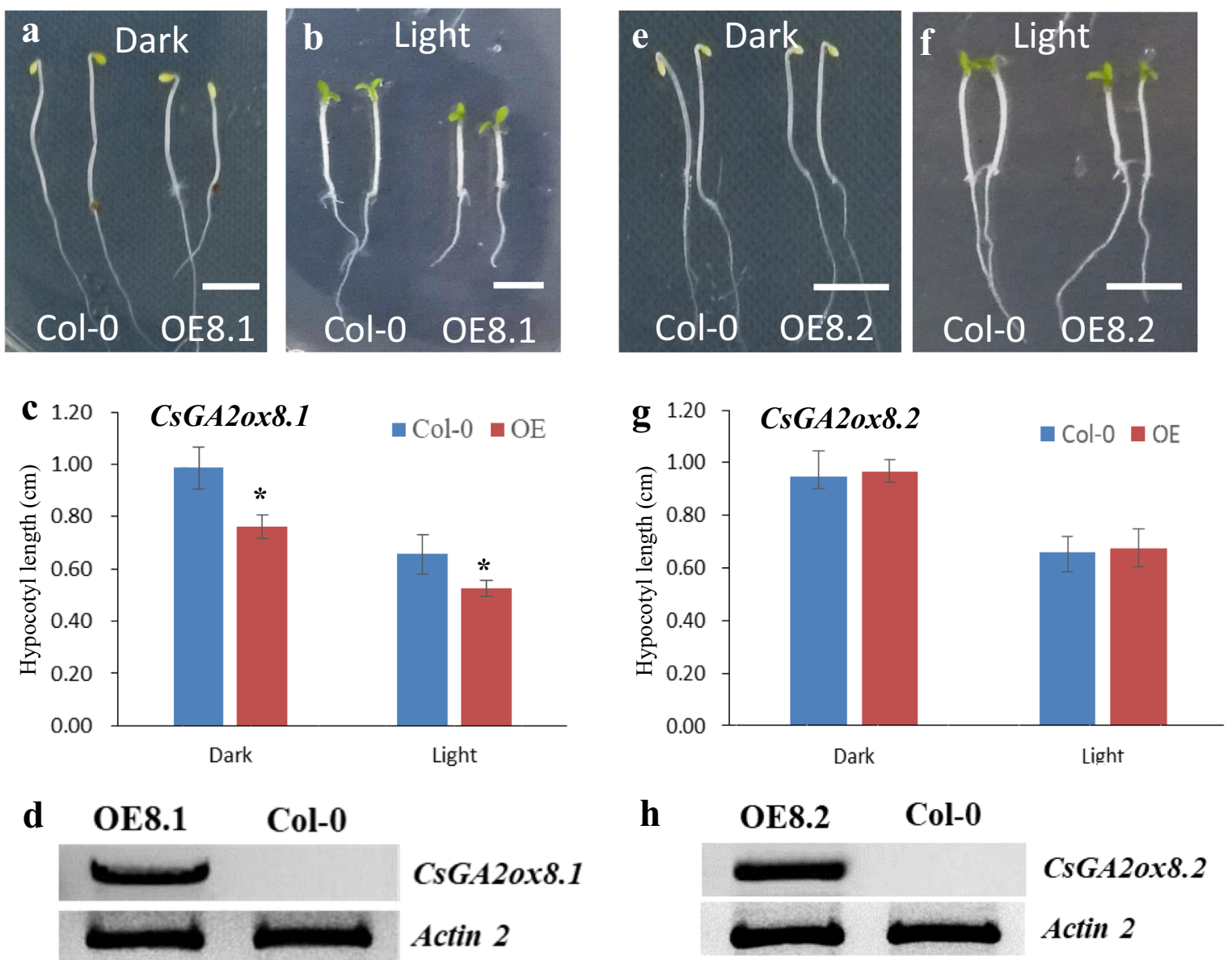

i
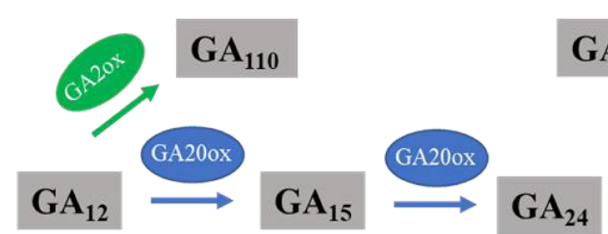

$\mathbf{G A}_{51}$
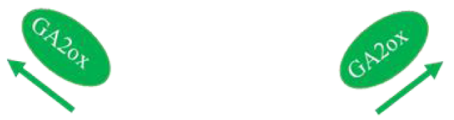

$\mathbf{G A}_{34}$

Col-0 18.66 [0.441]

OE8.1 $9.77^{\mathrm{b}}[0.430]$

OE8.2 18.89a [0.406]
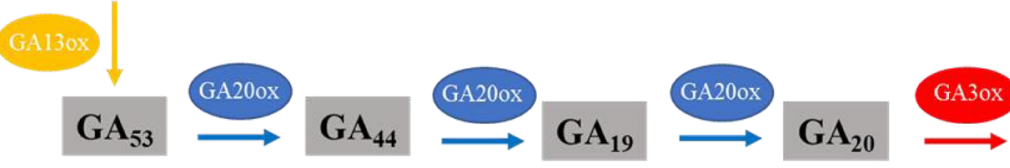

Col-0 6.58 a [0.392]

OE8.1 $2.25^{\mathrm{b}}[0.400]$

$0.17^{\mathrm{a}}[0.026]$

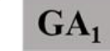

OE8.2 6.79a [0.582]

$0.07^{\mathrm{b}}[0.015]$

$0.10^{\mathrm{a}}[0.017]$

$0.16^{\mathrm{a}}[0.020]$

$0.02^{\mathrm{b}}[0.006]$

$0.11^{\mathrm{a}}[0.021]$

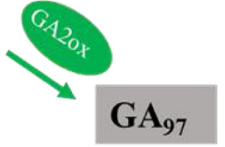

$\mathbf{G A}_{29}$

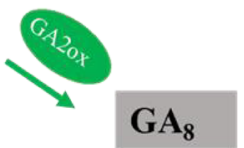


(see figure on previous page)

Fig. 3 CsGA2ox8.1 but not CsGA20x8.2 had a role in the deactivation of bioactive gibberellins (GAs). $\mathbf{a}$, b CsGA2ox8.1 overexpression (OE8.1) lines had shortened hypocotyls under both dark and light conditions. Scale bars, $5 \mathrm{~mm}$. c Hypocotyl lengths of the indicated genotypes in (a) and (b). The error bars indicate the standard deviations (SDs, $n>20$ ). The asterisks indicate significant differences between the hypocotyl lengths of OE8.1 lines and Col-0 $(P<0.05)$. d GA2Ox8.1 is overexpressed in OE8.1 but is not expressed in Col-0. e, $\mathbf{f}$ CsGA20x8.2 overexpression (OE8.2) lines had hypocotyl lengths similar to those of Col-0 under both dark and light conditions. $\mathbf{g}$ Hypocotyl lengths of the indicated genotypes in (e) and (f). The error bars indicate SDs $(n>20)$. $\mathbf{h}$ GA20x8.2 is overexpressed in OE8.2 but is not expressed in Col-0. i Profile of endogenous GAs in Col-0, OE8.1, and OE8.2 lines

a

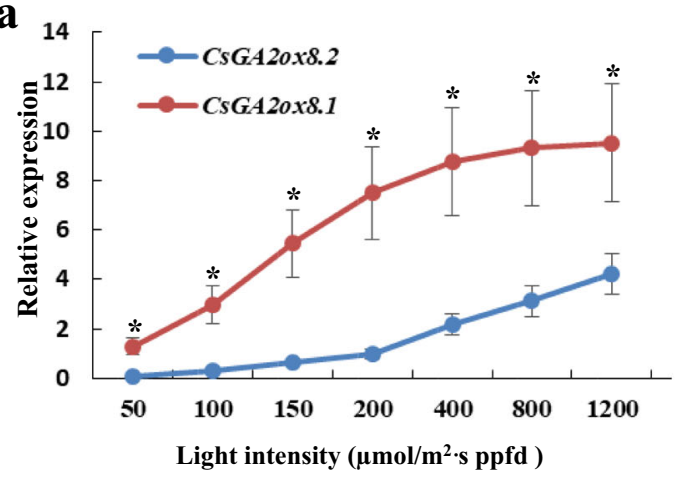

b

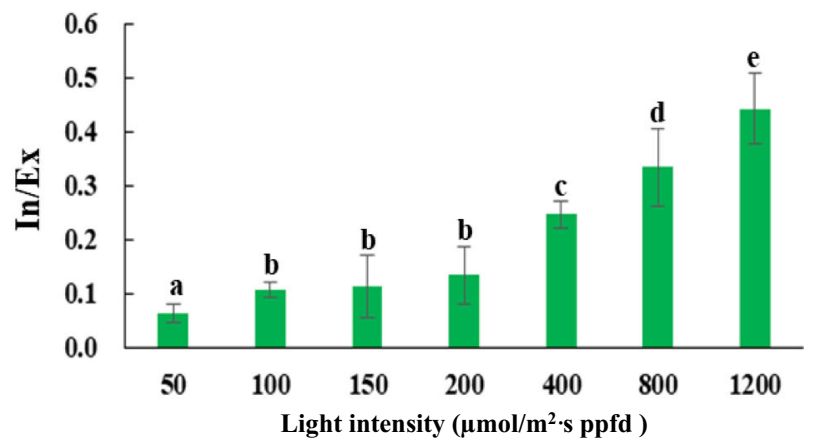

c

Light intensity

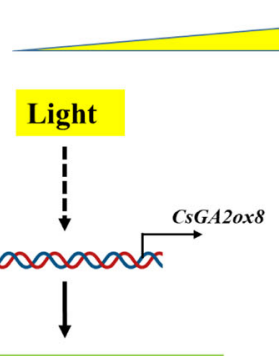

CsGA20x8.1

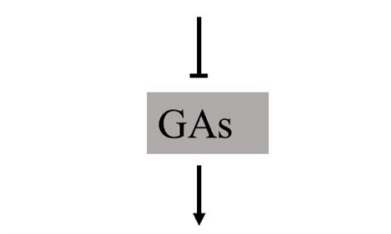

Hypocotyl elongation

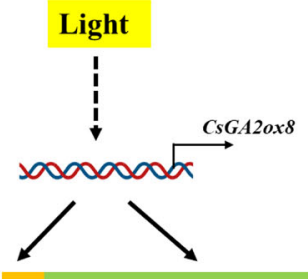

CSGA20x8.1

CsGA20x8.2

(No function)

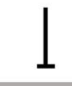

GAs

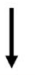

Hypocotyl elongation

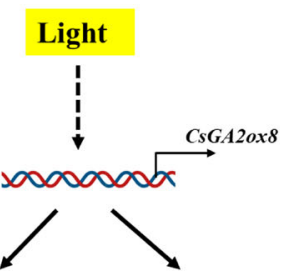

CsGA20x8.1

CsGA20x8.2

(No function)

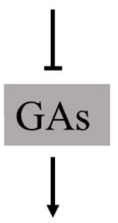

Hypocotyl elongation

\section{Hypocotyl Elongation}

Fig. 4 Plants tend to generate more CsGA20x8.2 but not CsGA2ox8.1 under high-light stress. a Relative expression levels of CsGA2Ox8.1 and CsGA20x8.2 under different light conditions as measured via quantitative reverse-transcription PCR (three replications). The asterisks indicate significant differences in gene expression between CsGA20x8.1 and CsGA20x8.2 $(P<0.05)$. $\mathbf{b}$ High light promotes the expression of CsGA20x8.2. The RNA products are quantified via the CsGA20x8.2/CsGA20x8.1 [inclusion/exclusion (In/Ex)] ratio. c Model depicting that plants buffer against high-light stress-induced functional CsGA20x8.1 via alternative splicing of a nonfunctional CsGA20x8.2 isoform to finely tune gibberellin (GA) levels and maintain hypocotyl elongation 
under low-light conditions, but under increasing light intensity, especially that corresponding to high-light stress, plants tended to generate more nonfunctional CsGA20x8.2 transcripts and limit the increase in functional CsGA2ox8.1 levels (Fig. 4c). In this way, plants could produce less functional GA2ox and could maintain a certain level of GA for hypocotyl elongation under highlight conditions.

AS is known to promote plant stress tolerance ${ }^{13}$. For example, stress imposed by high salinity promotes the use of noncanonical splice sites ${ }^{29}$. In addition, AS events finely tune gene expression by regulating the ratio of active and nonactive isoform ${ }^{30}$. Similarly, we showed that high-light stress increased the CsGA2ox8.2/CsGA2ox8.1 ratio in this study, suggesting a new mechanism for the GAdeactivating-enzyme response to high-light stress. However, it is not clear how high light modulates AS events. To our knowledge, AS events are mediated by core spliceosomal components (small nuclear ribonucleoproteins) as well as RNA-binding proteins (serine/arginine-rich proteins and heterogeneous nuclear ribonucleoproteins) ${ }^{30}$. One hypothesis is that high-light stress affects the expression or protein structure of spliceosome genes, thereby modulating AS events at the whole-genome level.

Our results suggest that IR may have been employed to finely tune gene expression by altering the ratio between functional and nonfunctional variants. Following a BLAST search of The Arabidopsis Information Resource (TAIR, https://www.arabidopsis.org/) and the MSU Rice Genome Annotation Project Database (http://rice. plantbiology.msu.edu/index.shtml), AT4G21200 and LOC_Os02g41954 were identified has sharing high sequence homology (57.42\% and $49.03 \%$, respectively) with that of CsGA2ox8. As shown in TAIR, AT4G21200 has one splice variant with an IR between the second and third exons, which is the same as CsGA2ox8.2, with the RI promoting the disruption of the open reading frame of AT4G21200, resulting in the generation of an mRNA with a premature stop codon (TGA), leading to a truncated protein $^{23}$. From the rice database, LOC_OsO2g41954 also has one splice variant, which has a premature stop codon (TGA) between the second and third exons ${ }^{30}$. These findings suggest that AS of GA2ox8 commonly occurs in plants, and this strategy might also be used by other plant species to buffer against the stress-responsive transcriptome and promote efficient adaptation to stress conditions.

\section{Materials and methods}

\section{Plant materials and growth conditions}

Cucumber inbred line 9930 was used in this study. Seeds were surface sterilized and soaked in water for $3 \mathrm{~h}$, after which they were then transferred to Petri dishes lined with wet filter papers and germinated at $28^{\circ} \mathrm{C}$.
The germinated seeds were then sown in autoclaved soil at $24^{\circ} \mathrm{C}$. Single cucumber seedlings with cotyledons emerging from the soil were chosen for light treatment. A. thaliana Col-0 was used in this study. The seeds were disinfected and germinated on Murashige-Skoog (MS) media consisting of $1 \%$ sucrose and $0.2 \%$ Phytagar at $4{ }^{\circ} \mathrm{C}$ for 3 days before being moved into a growth chamber at $22^{\circ} \mathrm{C}^{31}$.

\section{Light treatment and hypocotyl measurements}

Light intensities were measured using a Lighting Passport $^{\text {TM }}$ ALP-01 Handheld Spectrometer (HESON, Shanghai, China). Cucumber seedlings were grown in a greenhouse with "DE-HPS $1000 \mathrm{~W}$ " lights. To maintain the light conditions within an appropriate range, the plants were placed at different heights below the fixtures. For example, to generate a high-light condition, the plants were moved closer to the lamps; to generate low-light conditions, the plants were moved farther from the lamps or were covered with film. For Arabidopsis, the plates with disinfected seeds were treated at $4{ }^{\circ} \mathrm{C}$ for $72 \mathrm{~h}$ and then placed under white light for $10 \mathrm{~h}$ to induce uniform germination. Afterward, the plates were transferred to dark or light conditions and then incubated at $22^{\circ} \mathrm{C}$ for 3-6 days for hypocotyl measurements. The hypocotyl lengths of the seedlings were measured using a tapeline.

\section{GA level analysis}

The GA level was analyzed as previously reported, with moderate changes ${ }^{32}$. Briefly, for product purification, $\sim 0.5 \mathrm{~g}$ of the sample was quickly immersed in liquid nitrogen, homogenized, and extracted with $5 \mathrm{~mL}$ of precooled $80 \%$ methanol for $20 \mathrm{~h}$ at $4{ }^{\circ} \mathrm{C}$. Afterward, the samples were shaken at $4{ }^{\circ} \mathrm{C}$ for $30 \mathrm{~min}$ and then centrifuged at $18,000 \times g$ for $20 \mathrm{~min}$ at $4{ }^{\circ} \mathrm{C}$. The supernatant was collected and immersed in liquid nitrogen. Then, the samples were dissolved in $100 \mu \mathrm{L}$ of chromatographygrade methanol supplemented with $0.1 \mathrm{M}$ glacial acetic acid and filtered through a $0.22 \mu \mathrm{m}$ filter. Highperformance liquid chromatography-based analysis was performed using an Agilent (CA, USA) Zorbax SB- $\mathrm{C}_{18}$ column $(5 \mu \mathrm{m}, 4.6 \times 250 \mathrm{~mm})$. The mobile phase A solvent consisted of $100 \%$ methanol, and mobile phase $B$ was $0.1 \mathrm{M}$ acetic acid. The gradient elution was performed as follows: 0-40 min, 3\% A and 97\% B; after 40 min, $67.6 \% \mathrm{~A}$ and $32.2 \% \mathrm{~B}$. The flow rate was $1 \mathrm{~mL} \cdot \mathrm{min}^{-1}$, and the column temperature was set at $30^{\circ} \mathrm{C}$. Chromatograms were quantified using the Agilent chromatography workstation. Each sample involved three replicates from independent experiments.

\section{RNA-seq analysis and validation \\ Cucumber seedlings were subjected to two light conditions $\quad\left(\mathrm{X} 1, \quad 180 \mu \mathrm{mol} \cdot \mathrm{m}^{-2} \cdot \mathrm{s}^{-1} \quad \mathrm{PPFD} ; \quad \mathrm{X} 2\right.$,}


$40 \mu \mathrm{mol} \cdot \mathrm{m}^{-2} \cdot \mathrm{s}^{-1}$ PPFD) for $56 \mathrm{~h}$, after which the hypocotyls were harvested for RNA extraction (SV Total RNA Isolation System, Promega). Each treatment was replicated three times. The RNA quality was measured using an Agilent 2100 Bioanalyzer system (Agilent Technologies, CA, USA), with a RIN/RQ (RNA integrity number/ RNA quality index) $\geq 8$. Sequencing libraries were prepared using a NEBNext ${ }^{\circledR}$ Ultra $^{\text {TM }}$ RNA Library Prep Kit for Illumina ${ }^{\circledR}$ (NEB, USA). The libraries were sequenced on a HiSeq 4000 platform (Illumina, San Diego, CA). Thereafter, unknown or low-quality reads, as well as reads with adapters, were passed through a filter (SOAPnuke v1.5.2, https://github.com/BGI-flexlab/SOAPnuke) to generate clean reads ${ }^{33}$. The remaining high-quality clean reads were mapped to the Cucumber Reference Genome V3 version (ftp://cucurbitgenomics.org/pub/cucurbit/ genome/cucumber/Chinese_long/v3/3 ${ }^{34}$ using Bowtie2 v2.2.5. $5^{35}$. Differential expression analyses were subsequently carried out using RSEM v1.2.12 ${ }^{36}$ software. Differentially expressed genes were defined as those having an $\mid \log 2$ fold change (FC) $\mid \geq 1$ and a $q$-value $\leq 0.05$. DAS analyses were performed using rMATS v3.2.5 $5^{37}$. Transcripts were deemed to be the result of DAS when the false discovery rate was $\leq 0.05$.

To validate the GA2ox8 AS isoforms, seedlings were treated with the same light conditions as those applied for RNA-seq (X1, $\quad 180 \mu \mathrm{mol} \cdot \mathrm{m}^{-2} \cdot \mathrm{s}^{-1} \quad$ PPFD; X2, $\left.40 \mu \mathrm{mol} \cdot \mathrm{m}^{-2} \cdot \mathrm{s}^{-1} \mathrm{PPFD}\right)$ for $56 \mathrm{~h}$, after which the hypocotyls were harvested for RNA extraction, cDNA synthesis, and PCR-based analysis. The primers used are listed in Supplementary Table 3.

\section{Sequence alignment}

Sequence alignments were performed based on the amino acid sequences of CsGA2ox8.1, CsGA2ox8.2, and AtGA2ox8. Multiple sequence alignment was performed using DNAMAN for Windows (Lynnon Corporation, Quebec).

\section{Gene expression analysis}

Gene expression analysis methods were used as previously reported ${ }^{31}$. Total RNA was extracted from the hypocotyls using an SV Total RNA Isolation System (Promega), and cDNA was synthesized using a MultiScribe ${ }^{\mathrm{TM}}$ Reverse Transcriptase Kit (Applied Biosystems) according to the manufacturer's instructions. Quantitative RT-PCR was performed using a SYBR ${ }^{\circledR}$ Premix Ex Taq ${ }^{\mathrm{TM}}$ Kit (TaKaRa, China) in conjunction with an Applied Biosystems 7500 Real-time PCR system. For RT-PCR, PCR primers were designed to amplify two isoforms of different sizes, and their RNA products were quantified and reported as an In/Ex ratio ${ }^{38}$. Cucumber $\alpha$-TUBULIN (TUA) and Arabidopsis ACTIN 2 were used as internal controls $^{39}$. The primers used are listed in Supplementary Table S3.

\section{Transformation of $A$. thaliana}

Transformation assays were performed as previously described $^{31}$. To generate CsGA2ox8.1 and CsGA2ox8.2 overexpression constructs, full-length CsGA2ox8.1 and CsGA2ox8.2 cDNAs were cloned and inserted into a pBI121 vector between the XbaI and SmaI restriction sites ${ }^{40}$. The constructs were then introduced into Agrobacterium tumefaciens strain LBA4404 by electroporation, which were then transformed into wild-type $A$. thaliana (Col-0), as previously described ${ }^{41}$. The transgenic plants were screened on MS media supplemented with $50 \mathrm{mg} / \mathrm{L}$ kanamycin and then confirmed via PCR. The primers used are listed in Supplementary Table S3.

\section{Statistical analysis}

All the treatments mentioned in this study involved at least three independent biological and technical replicates. The results were analyzed using analysis of variance. All the analyses were carried out using Statistics Analysis System 15.1 for Windows.

\section{Acknowledgements \\ This study was supported by The National Key Research and Development Program of China (2019YFD1000300), the International Postdoctoral Exchange Fellowship Program from the China Postdoctoral Council (20170053), the Technology System Construction of Modern Agricultural Industry of Shanghai (19Z113040008), and the Presidential Foundation of Guangdong Academy of Agricultural Sciences (BZ201901). \\ Author details \\ 'School of Agriculture and Biology, Shanghai Jiao Tong University, Key Laboratory of Urban Agriculture (South), Ministry of Agriculture, Dongchuan Road, Shanghai 200240, China. '2Department of Plant Genomics, Centre for Research in Agricultural Genomics (CRAG), CSIC-IRTA-UAB-UB, Bellaterra 08193, Spain. ${ }^{3}$ Vegetable Research Institute, Guangdong Academy of Agricultural Sciences, Guangzhou 510640, China. ${ }^{4}$ Guangdong Key Laboratory for New Technology Research of Vegetables, Guangzhou 510640, China}

\section{Author contributions}

D.H. and B.L. proposed the project; B.L. and J.Y. designed the research; B.L., J.Y. S.Z., Y.Y., and P.L. performed the experiments; and B.L. and J.Y. analyzed the data and wrote the paper, with the participation of Q.N. and D.H.

Conflict of interest

The authors declare that they have no conflict of interest.

Supplementary Information accompanies this paper at (https://doi.org/ 10.1038/s41438-020-00430-w).

Received: 12 May 2020 Revised: 13 September 2020 Accepted: 17 October 2020

Published online: 01 January 2021

\section{References}

1. Lopez-Juez, E., Kobayashi, M., Sakurai, A., Kamiya, Y. \& Kendrick, R. E. Phytochrome, gibberellins, and hypocotyl growth (A study using the cucumber (Cucumis sativus L.) long hypocotyl mutant). Plant Physiol. 107, 131-140 (1995). 
2. Sun T-p. The molecular mechanism and evolution of the GA-GID1-DELLA signaling module in plants. Curr. Biol. 21, R338-R345 (2011).

3. Griffiths, J. et al. Genetic characterization and functional analysis of the GID1 gibberellin receptors in Arabidopsis. Plant Cell 18, 3399-3414 (2006).

4. Cheng, J. et al. A single nucleotide mutation in GID1C disrupts its interaction with DELLA1 and causes a GA-insensitive dwarf phenotype in peach. Plant Biotechnol. J. 17, 1723-1735 (2019).

5. Zhao, B. et al. Brassica napus DS-3, encoding a DELLA protein, negatively regulates stem elongation through gibberellin signaling pathway. Theor. Appl. Genet. 130, 727-741 (2017).

6. Kami, C., Lorrain, S., Hornitschek, P., \& Fankhauser, C. in Current Topics in Developmental Biology (Elsevier, 2010).

7. García-Martinez, J. L. \& Gil, J. Light regulation of gibberellin biosynthesis and mode of action. J. Plant Growth Regul. 20, 354-368 (2002).

8. Gawronska, $H$. et al. Effects of low irradiance stress on gibberellin levels in pea seedlings. Plant Cell Physiol. 36, 1361-1367 (1995).

9. De Lucas, M. et al. A molecular framework for light and gibberellin control of cell elongation. Nature 451, 480-484 (2008).

10. Feng, $\mathrm{S}$. et al. Coordinated regulation of Arabidopsis thaliana development by light and gibberellins. Nature 451, 475-479 (2008).

11. Li, K. et al. DELLA-mediated PIF degradation contributes to coordination of light and gibberellin signalling in Arabidopsis. Nat. Commun. 7, 1-11 (2016).

12. Colebrook, E. H., Thomas, S. G., Phillips, A. L. \& Hedden, P. The role of gibberellin signalling in plant responses to abiotic stress. J. Exp. Biol. 217, 67-75 (2014).

13. Laloum, T., Martín, G. \& Duque, P. Alternative splicing control of abiotic stress responses. Trends Plant Sci. 23, 140-150 (2018).

14. Chaudhary, S., Jabre, I., Reddy, A. S., Staiger, D. \& Syed, N. H. Perspective on alternative splicing and proteome complexity in plants. Trends Plant Sci. 24 496-506 (2019).

15. Schomburg, F. M., Bizzell, C. M., Lee, D. J., Zeevaart, J. A. \& Amasino, R. M. Overexpression of a novel class of gibberellin 2-oxidases decreases gibberellin levels and creates dwarf plants. Plant Cell 15, 151-163 (2003).

16. Busov, V. B. et al. Activation tagging of a dominant gibberellin catabolism gene (GA 2-oxidase) from poplar that regulates tree stature. Plant Physiol. 132, 1283-1291 (2003)

17. Huang, J. et al. Activation of gibberellin 2-oxidase 6 decreases active gibberellin levels and creates a dominant semi-dwarf phenotype in rice (Oryza sativa L.). J. Genet. Genomics 37, 23-36 (2010).

18. Otani, M. et al. Overexpression of the gibberellin 2-oxidase gene from Torenia fournieri induces dwarf phenotypes in the liliaceous monocotyledon Tricyrtis sp. J. Plant Physiol. 170, 1416-1423 (2013).

19. Wuddineh, W. A. et al. Identification and overexpression of gibberellin 2oxidase (GA 2ox) in switchgrass (Panicum virgatum L.) for improved plant architecture and reduced biomass recalcitrance. Plant Biotechnol. J. 13, 636-647 (2015)

20. Hu, Y.-X., Tao, Y.-B. \& Xu, Z.-F. Overexpression of Jatropha Gibberellin 2-oxidase 6 (JcGA20x6) induces dwarfism and smaller leaves, flowers and fruits in Arabidopsis and Jatropha. Front. Plant Sci. 8, 2103 (2017).

21. Chaudhary, $\mathbf{S}$. et al. Alternative splicing and protein diversity: plants versus animals. Front. Plant Sci. 10, 708 (2019).
22. Filichkin, S. A. et al. Genome-wide mapping of alternative splicing in Arabidopsis thaliana. Genome Res. 20, 45-58 (2010).

23. Marquez, Y., Brown, J. W., Simpson, C., Barta, A. \& Kalyna, M. Transcriptome survey reveals increased complexity of the alternative splicing landscape in Arabidopsis. Genome Res. 22, 1184-1195 (2012).

24. Plackett, A. R. et al. Analysis of the developmental roles of the Arabidopsis gibberellin 20-oxidases demonstrates that GA200x1, -2, and -3 are the dominant paralogs. Plant Cell 24, 941-960 (2012).

25. Hedden, P. \& Proebsting, W. M. Genetic analysis of gibberellin biosynthesis. Plant Physiol. 119, 365-370 (1999).

26. Wang, G.-L. et al. Morphological characteristics, anatomical structure, and gene expression: novel insights into gibberellin biosynthesis and perception during carrot growth and development. Horticulture Res. 2, 1-10 (2015).

27. Filichkin, S. A. et al. Environmental stresses modulate abundance and timing of alternatively spliced circadian transcripts in Arabidopsis. Mol. Plant 8, 207-227 (2015).

28. Kalyna, M. et al. Alternative splicing and nonsense-mediated decay modulate expression of important regulatory genes in Arabidopsis. Nucleic Acids Res. 40 2454-2469 (2012).

29. Feng, J. et al. SKIP confers osmotic tolerance during salt stress by controlling alternative gene splicing in Arabidopsis. Mol. Plant 8, 1038-1052 (2015).

30. Kawahara, Y. et al. Improvement of the Oryza sativa Nipponbare reference genome using next generation sequence and optical map data. Rice 6, 4 (2013).

31. Liu, B. et al. Silencing of the gibberellin receptor homolog, CsGID1a, affects locule formation in cucumber (Cucumis sativus) fruit. N. Phytologist 210, 551-563 (2016).

32. Yang, R. et al. Hormone profiling and transcription analysis reveal a major role of ABA in tomato salt tolerance. Plant Physiol. Biochem. 77, 23-34 (2014).

33. Li, R. et al. SOAP2: an improved ultrafast tool for short read alignment. Bioinformatics 25, 1966-1967 (2009).

34. Li, Q. et al. A chromosome-scale genome assembly of cucumber (Cucumis sativus L.). GigaScience $\mathbf{8}$, giz072 (2019).

35. Langmead, B. \& Salzberg, S. L. Fast gapped-read alignment with Bowtie 2. Nat Methods 9, 357 (2012)

36. Li, B. \& Dewey, C. N. RSEM: accurate transcript quantification from RNA-Seq data with or without a reference genome. BMC Bioinforma. 12, 323 (2011).

37. Shen, S. et al. rMATS: robust and flexible detection of differential alternative splicing from replicate RNA-Seq data. Proc. Natl Acad. Sci. USA 111, E5593-E5601 (2014).

38. Zhou, X. et al. Transcriptome analysis of alternative splicing events regulated by SRSF10 reveals position-dependent splicing modulation. Nucleic Acids Res. 42, 4019-4030 (2014).

39. Zhang, Y. et al. A GAMYB homologue CsGAMYB1 regulates sex expression of cucumber via an ethylene-independent pathway. J. Exp. Bot. 65, 3201-3213 (2014).

40. Jefferson, A. R. Assaying chimeric genes in plants: the GUS gene fusion system. Plant Mol. Biol. Report. 5, 387-405 (1987).

41. Clough, S. J. \& Bent, A. F. Floral dip: a simplified method for Agrobacterium-mediated transformation of Arabidopsis thaliana. Plant J. 16 735-743 (1998). 